\title{
An AC-type element mediates transactivation of secondary cell wall carbohydrate-active enzymes by PttMYB021, the Populus MYB46 orthologue
}

\author{
Ines Ezcurra*', Camilla Johansson, Prashanth Tamizhselvan, Anders Winzell, Henrik Aspeborg \\ From IUFRO Tree Biotechnology Conference 2011: From Genomes to Integration and Delivery \\ Arraial d'Ajuda, Bahia, Brazil. 26 June - 2 July 2011
}

\section{Background}

The transcription factor MYB46, together with its redundant paralog MYB83, regulates expression of secondary cell wall biosynthesis genes in Arabidopsis [reviewed in 1].

We isolated the Populus MYB46 ortholog, PtxtMYB021 from hybrid aspen (Populus tremula $x$ tremuloides) [2]. Transiently expressed MYB021 transactivated gene promoters of Populus xylan-active enzymes GT43A, GT43B and Xyn10A. Analysis of conserved motifs within these promoters identified the sequence CCACCAAC, which is similar to the $\mathrm{AC}$ elements mediating transactivation by MYB transcription factors during lignin biosynthesis, and we showed that this motif is enriched in xylem-specific carbohydrate active enzyme (CAZyme) promoters.

\section{Methods}

To establish whether the AC-type motif in CAZyme promoters is important for their function, we analyzed loss-of-function and gain-of-function GUS reporter constructs of the GT43A promoter in a transient transcription assay, by co-infiltration with a 35S:MYB021 effector in Nicotiana benthamiana leaves.

\section{Results and discussion}

We show that mutation of the AC-type element in the GT43A promoter abolishes transactivation by MYB021, whereas an AC multimer confers MYB021 transactivation to a minimal $35 \mathrm{~S}$ promoter. Others have shown that the recombinant Eucalyptus grandii MYB46/MYB83 ortholog, EgMYB2, directly binds the AC-motif variant CCACCTACC found in the EgCCR gene promoter [3],

\footnotetext{
* Correspondence: ines@biotech.kth.se

KTH Biotechnology, 10691 Stockholm, Sweden

Full list of author information is available at the end of the article
}

suggesting that MYB021 directly binds the AC-type motif. We propose that, in angiosperms, the AC-type regulatory element mediates MYB46's transactivation of its target genes. Further, MYB46 activates lignocellulose synthesis in a feed-forward loop with downstream MYBs, involving both direct activation of downstream MYBs and joint regulation of downstream secondary cell wall synthesis targets (Fig. 1). Recent results show

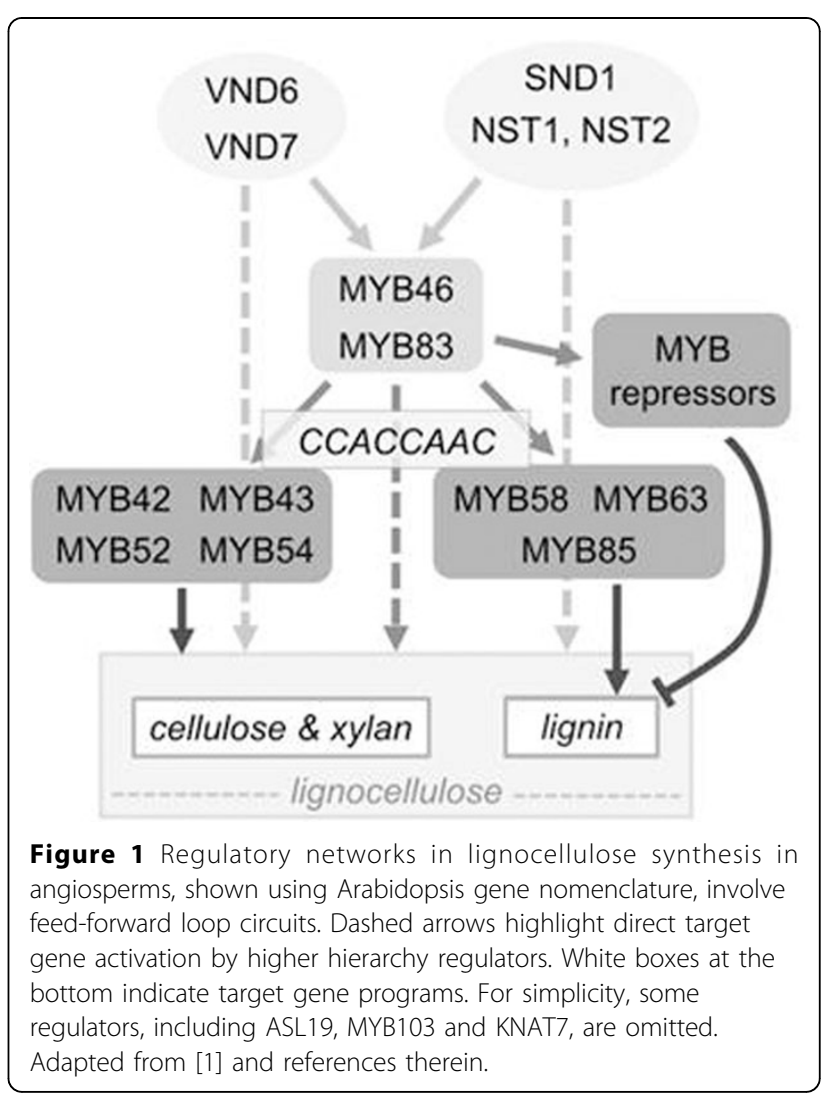


Table 1 Sequence overlap in AC motifs.

\begin{tabular}{|c|c|c|c|c|c|}
\hline Species & Genbe & Motif name & Motif sequence & MYB protein ${ }^{\dagger}$ & Reference \\
\hline Phaseolus & PAL2 & AC-I & CCCACCTACC & n.d. & [9] \\
\hline Phaseolus & PAL2 & AC-II & CCACCAACCC... & n.d. & [9] \\
\hline Petroselinum & $4 C L 1$ & AC-II & CTCACCAACCC & n.d. & [10] \\
\hline Populus & CCOAOMT & AC-II & CTCACCAACCC... & n.d. & [7] \\
\hline Eucalyptus & $\mathrm{EgCCR}$ & MBSIIG & cСACCTACC & EgMYB2 $^{\S}$ & [3] \\
\hline Eucalyptus & $\mathrm{EgCCR}$ & MBSIIG & cСACCTACC & EgMYB1 & [11] \\
\hline Arabidopsis & $4 C L 1$ & AC-II & tCACCAAC & MYB58/63 & [6] \\
\hline Zea & ZmCOMT & AC-II & tcACCAAC & ZmMYB31 & [12] \\
\hline Populus & GT43A & AC-type & $\frac{\text { CCACCAAC }}{* * * * * *}$ & PttMYB021 $1^{\S}$ & [2] \\
\hline
\end{tabular}

In motif sequences, lowercase indictates ases outside the defined motif, and asterisks highlight invariant bases. Similarity to the GT43A AC-type elements is underlined. ${ }^{+}$Indicates which MYB protein binds the motif. ${ }^{\S}$ Ortholog of MYB46.

direct activation of downstream lignocellulose synthesis targets by higher hierarchy NAC domain regulators $[4,5]$.

Interestingly, the downstream MYB regulators of lignin biosynthesis MYB58 and MYB63 bind the AC element but reportedly do not regulate CAZyme-mediated secondary cell wall polysaccharide biosynthesis [6]. Other downstream MYBs that bind variants of the AC element are the EAR domain lignin repressors, such as EgMYB1 and ZmMYB31 (Table 1). Then, binding of the distinct lignocellulose synthesis MYB transcription factors to particular AC-element variants may be mediated by motif sequence, motif context and/or interactions with cofactors. Also, some AC element variants overlap extensively with each other and with the AC-type element defined by us (Table 1), so it remains to be established whether they are all representatives of one identical core sequence motif, as previously proposed [7]. Establishing the exact sequence requirements for AC-element recognition by distinct MYBs will facilitate elucidation of lignocellulose-related regulatory networks in different species, through bioinformatic analysis [8].

\section{Conclusions}

The AC regulatory element, here preliminarily redefined as the sequence CCACCAAC, is a true "lignocellulose synthesis response element", mediating MYB46-dependent transactivation of the whole secondary cell wall gene program. This knowledge will enable inference of MYB46centered regulatory networks in different species, through bioinformatic analysis.

\section{Acknowledgements}

We thank David Caparrós-Ruiz for helpful comments and discussions.
References

1. Ohashi-lto K, Fukuda $\mathrm{H}$ : Transcriptional regulation of vascular cell fates. Curr Opin Plant Biol 2010, 13:670-676.

2. Winzell A, Aspeborg $H$, Wang $Y$, Ezcurra I: Conserved CA-rich motifs in gene promoters of PtxtMYB021-responsive secondary cell wall carbohydrate-active enzymes in Populus. Biochem Biophys Res Commun 2010, 394:848-853.

3. Goicoechea M, Lacombe E, Legay S, Mihaljevic S, Rech P, et al: EgMYB2, a new transcriptional activator from Eucalyptus xylem, regulates secondary cell wall formation and lignin biosynthesis. Plant J 2005, 43:553-567.

4. Zhong R, Lee C, Ye ZH: Global analysis of direct targets of secondary wall NAC master switches in Arabidopsis. Mol Plant 2010, 3:1087-1103.

5. Yamaguchi M, Mitsuda N, Ohtani M, Ohme-Takagi M, Kato K, Demura T: VASCULAR-RELATED NAC-DOMAIN 7 directly regulates the expression of a broad range of genes for xylem vessel formation. Plant J 2011.

6. Zhou J, Lee C, Zhong R, Ye ZH: MYB58 and MYB63 are transcriptional activators of the lignin biosynthetic pathway during secondary cell wall formation in Arabidopsis. Plant Cell 2009, 21:248-266.

7. Raes J, Rohde A, Christensen JH, Van de Peer Y, Boerjan W: Genome-wide characterization of the lignification toolbox in Arabidopsis. Plant Physiol 2003, 133:1051-1071.

8. Pilpel $Y$, Sudarsanam $P$, Church GM: Identifying regulatory networks by combinatorial analysis of promoter elements. Nat Genet 2001, 29:153-159.

9. Hatton D, Sablowski R, Yung MH, Smith C, Schuch W, Bevan M: Two classes of cis sequences contribute to tissue-specific expression of a PAL2 promoter in transgenic tobacco. Plant J 1995, 7:859-876.

10. Hauffe KD, Lee SP, Subramaniam R, Douglas CJ: Combinatorial interactions between positive and negative cis-acting elements control spatial patterns of 4CL-1 expression in transgenic tobacco. Plant J 1993, 4:235-253.

11. Legay S, Sivadon P, Blervacq AS, Pavy N, Baghdady A, et al: EgMYB1, an R2R3 MYB transcription factor from eucalyptus negatively regulates secondary cell wall formation in Arabidopsis and poplar. New Phytol 2010, 188:774-786.

12. Fornalé $S$, Shi $X$, Chai $C$, Encina $A$, Irar $S$, et al: ZmMYB31 directly represses maize lignin genes and redirects the phenylpropanoid metabolic flux. Plant J 2010, 64:633-644.

doi:10.1186/1753-6561-5-S7-040

Cite this article as: Ezcurra et al:: An AC-type element mediates transactivation of secondary cell wall carbohydrate-active enzymes by PttMYB021, the Populus MYB46 orthologue. BMC Proceedings 20115

(Suppl 7):040. 